\title{
Commentaire de la lettre à la rédaction de Max Goyffon « La pénurie de sérums antivenimeux »
}

\author{
G. Mion · S. Larréché \\ Reçu le 26 décembre 2015 ; accepté le 26 décembre 2015 \\ (C) Société de pathologie exotique et Lavoisier SAS 2016
}

Nous avons lu avec beaucoup d'intérêt la lettre de Max Goyffon [2] qui expose le problème terrible de la pénurie d'antivenins dans le monde et des dizaines de milliers de morts évitables qu'elle entraine, indépendamment de l'arrêt annoncé du FAV-Afrique. Tout en abondant dans le sens du Pr Goyffon, nous aimerions ajouter quelques commentaires.

Il est vrai que dans certains cas d'envenimations ophidiennes, deux à quatre ampoules sont parfois indispensables, mais dans notre expérience des morsures d'Echis pyramidum à Djibouti, une seule ampoule s'est révélée le plus souvent suffisante en particulier lorsque le patient pris en charge est au stade de la coagulopathie sans saignement associé [4,5]. Or le genre Echis est responsable de l'essentiel des décès en Afrique. Une utilisation rationnelle des antivenins est un préalable nécessaire afin d'éviter un gaspillage de ces médicaments indispensables. Dans cette optique, les études cliniques s'intéressant à l'optimisation des protocoles d'utilisation des antivenins doivent être encouragées.

Le chiffre de 7000 décès annuels en Afrique est d'ailleurs peut-être à nuancer. L'approche méta-analytique de Chippaux (avec les limites inhérentes aux méta-analyses) propose une fourchette de 5000 à 9500 décès annuels [1] Gutierrez et al avançaient en 2010 des chiffres trois fois plus élevés [3]. En fait, personne ne sait avec exactitude combien de décès annuels on peut imputer aux morsures de serpents. La réalité se situe peut-être dans la fourchette de 7000 à 20000 décès.

Enfin, le fait que seules $3 \%$ des victimes soient traitées pose un problème de fond : d'une part, on peut penser que ce chiffre catastrophique et qui ne s'améliore pas en dépit de

\section{G. Mion $(\bowtie)$}

Département d'anesthésie-réanimation, Hôpital Cochin,

27, rue du Faubourg Saint-Jacques,

75014 Paris, France

e-mail : georges-mion@bbox.fr

\section{S. Larréché}

Service de biologie médicale,

Hôpital d'instruction des armées Bégin,

69, avenue de Paris, 94160 Saint-Mandé, France décennies d'alertes lancées par les experts, est la conséquence directe du prix et de la rareté de l'antivenin. L'inverse est peut-être également vrai : s'il était plus souvent prescrit, le traitement antivenimeux serait plus souvent disponible et moins onéreux. D'autre part, on sait qu'il est surtout la conséquence d'un système de soins inadapté (Max Goyffon le rappelle clairement) avec une pénurie de personnels formés, une absence de protocoles validés, mais aussi le fait du passage quasi-obligatoire par les tradipraticiens. Ainsi, cette proportion ridicule de malades traités amoindrit la catastrophe annoncée : malheureusement, la disparition du FAV-Afrique n'affectera que cette petite proportion des $3 \%$ traités.

C'est la raison pour laquelle il faudrait effectivement mettre en parallèle cette problématique de pénurie d'antivenin, annoncée et dénoncée, avec une encore plus problématique organisation (ou plutôt désorganisation) de beaucoup de systèmes de santé africains. Les causes en sont connues et récurrentes : calculs politiciens, luttes de pouvoir ou luttes claniques, voire détournements financiers. Le financement des programmes de lutte contre les envenimations n'est bien entendu pas le moindre de ces problèmes.

Liens d'intérêts : les auteurs déclarent ne pas avoir de liens d'intérêts.

\section{Références}

1. Chippaux JP (2011) Estimate of the burden of snakebites in sub-Saharan Africa: a meta-analytic approach. Toxicon 57(4): 586-99

2. Goyffon M (2016) La pénurie de sérums antivenimeux. Bull Soc Pathol Exot 108(5)

3. Gutiérrez JM, Williams D, Fan HW, et al (2010) Snakebite envenoming from a global perspective: Towards an integrated approach. 56(7):1223-35

4. Larréché S, Mion G, Mayet A, et al (2011) Antivenin remains effective against African Viperidae bites despite a delayed treatment. Am J Emerg Med 29(2):155-61

5. Mion G, Larréché S, Benois A, et al (2013) Dynamics of hemostasis during coagulopathy resulting from Echis envenomation. Toxicon 76:103-9 